\title{
Magnetic Refrigeration: A Promising Substitute for Vapour Compression System
}

\author{
ArchanaAnsolia \\ Department of mechanical Engineering, Maulana Azad National Institute of Technology, Bhopal, India \\ Dr. Manoj Arya \\ Department of mechanical Engineering, Maulana Azad National Institute of Technology, Bhopal, India
}

\begin{abstract}
In this paper a new type of refrigeration technology, Magnetic Refrigeration has been discussed. The objective of this paper is to explain the principle, construction, working and the operating cycles of Magnetic Refrigerator. The development of this system leads to improve the COP and to reduce Ozone layer Depletion and Global Warming. The main principle behind this refrigeration system is Magneto-Caloric Effect (MCE). According to this effect whenever some magnetic materials like Gadolinium have subjected to a magnetic field the temperature of that material increases. Whenever the magnetic field has removed the temperature of that material diminishes down again. This is because of the randomization and alignment of atoms in the magnetic material. The magnetic refrigerator uses the Magneto-Caloric Effect in the following way. Gadolinium is arranged to pass through a magnetic field. As it passes through the field, the gadolinium heats up as it exhibits magneto caloric effect. If it is taken out of the field then it comes down to the original temperature. But we will circulate the water to draw the heat out of the metal when it is in the magnetic field. As the material leaves the magnetic field, the material cools to a temperature much below the original temperature as a result of the magneto caloric effect. Then this cold Gadolinium is used to remove the heat from the refrigerator's coils.
\end{abstract}

Keywords: Magnetic refrigeration, magneto-caloric effect, magnetic materials, magnetic refrigeration cycle

\section{INTRODUCTION}

The vapor-compression cycle is a well-established refrigeration technique used in most household refrigerators, airconditioners, and many large commercial and industrial refrigeration systems. However, mankind is facing serious problems concerning the global environment, and the emissions of conventional refrigerant fluids such as a chlorofluorocarbon(CFC), hydro chlorofluorocarbon(HCFC) and hydro fluorocarbon(HFC) are responsible for ozone depletion or the green house effect. From the view point of energy consumption, refrigeration systems have a large impact on total power consumption. Nowadays, there search and development of environmentally friendly and efficient new refrigeration technology is urgently required.

Refrigeration based on the magneto-caloric effect is a modern way to decrease temperature. This method is environmentally friendly because, unlike traditional technology which consists of expansion and compression of gasses, it does not employ chlorofluorocarbon gasses which contribute to the greenhouse effect. Moreover, magnetic refrigerators are characterized by a higher efficiency compared to commonly used cooling appliances (13).

The future of magnetic refrigeration looks bright. Much research is ongoing in the field of magneto-caloric materials and magnetic refrigeration systems to find a suitable way to make an efficient machine. Even though significant advances are achieved in giant magneto -caloric materials around room temperature and the feasibility of magnetic cooling technology has been demonstrated through several prototypes [1,4], more work on both the fundamental and practical plane is needed to master this technology and introduce it in industrial and domestic applications.

Magnetic refrigeration (MR) is based on the magneto-caloric effect (MCE), defined as an intrinsic property of some magnetic materials which respond to an external applied magnetic field by a variation in their temperature. The temperature increases when the magnetic field is applied (magnetization phase) and decreases when the magnetic field is removed (demagnetization phase). This shows the fundamental and important role of the applied magnetic field. Thus, the design of the magnetic field source in MR systems should be one of the first issues that researchers should focus on. On the international level, many teams are working on new MR systems structures. Particular efforts have been done to increase magnetic induction for more powerful MR systems. 
This technology was successfully applied in low-temperature physics since the 1930's to cool down samples from a few Kelvin to a few hundred of a Kelvin above the absolute zero point $\quad$ (-273.15 K). But today, because of two important aspects, also applications for the refrigeration market seem feasible. The first one is the availability of magneto caloric materials with Curie temperatures at room temperature and above. Furthermore, by the "giant" magneto caloric effect (see below) new magneto caloric materials have become a factor two to three more performing.

Because this article shall only give an overview on the technology of magnetic refrigeration written for non-specialists, readers with a deeper interest are referred to some comprehensive review articles.

\section{LITERATURE REVIEW}

It is well known that the efficiency of the conventional gas compression/ expansion refrigeration system cannot significantly be improved. Also, for the conventional refrigeration system, there exist serious concerns for the environment. Thus, it is necessary and important to explore other alternative cooling technologies. The magnetic refrigerator, which has advantages in refrigeration efficiency, reliability, low noise and environmental friendliness with respect to the conventional gas refrigerators, is becoming a promising technology to replace the conventional technique.

The study of magnetic refrigeration was started with the discovery of magneto-caloric effect (MCE) 132 years ago. It was first discovered by Warburg in 1881 in iron by German physicist "Emil Warburg". Usually, the temperature increases when the field is applied and decreases when the field is removed and the process is reversible. In1890 Tesla and in 1892 Edison independently and unsuccessfully tried to benefit from this effect by running heat engines. In1918 Weissand Piccard explained the magneto-caloric effect. Later Debye and Giauque proposed a method of magnetic refrigeration for low-temperature physics in order to obtain sub-Kelvin temperatures. In1933 Giauque and MacDougall successfully verified the method by experiment. Then it has been used in cryogenic refrigeration since the 1930s. It is maturely used in liquefaction of hydrogen and helium. In 1976, at Lewis Research Center of American National Aeronautics and Space Administration, Brown first applied the magnetic refrigeration in a room-temperature range.

The first "room temperature magnetic refrigerator" containing permanent magnets was designed and built in 2001 in the USA by the Astronautics Corporation. The early prototypes were able to reach high magnetic flux densities in the magneto-caloric material only if superconducting magnets were applied. Research today is focused on improvements of magneto-caloric materials, magnets and an optimal design of magnetic refrigerator devices for room temperature applications.

Z. Chen. in 2003 explain the concept if MCE, its development of magnetic material. Magnetic Refrigeration cycles and some prototype are reviewed. D Eriksen, in 2013 explains A novel Rotary magnetic refrigeration device has been designed and constructed. H. R. E. H. Bouchekara and A. Kedous, in 2013, explain in his paper "computational algorithms of different multi-objective optimization techniques and their application for AMR systems are presented. The results obtained using some methods, like the weighting methods, are properly balanced yielding the best compromise in the presence of conflicting objectives while some other methods, like the distance to a reference goal method and the $\varepsilon$-constraint method, allow to reach predefined goals or to respect additional constraints. Moreover, methods like distance to a reference one or the $\varepsilon$-constraint one need some expertise and insights". Y. I. Spichkin, R. R. Gimaev, in 2013, explain in his paper "The methods of experimental investigations of the magneto-caloric parameters are reviewed and discussed. A.Czernuszewicz, J. Kaleta, M. Kro'lewicz, D. Lewandowski, in 2013, explain in his paper “This paper presents a laboratory test stand for magnetocaloric effect investigations in materials with transition temperatures close to room temperature. A cylindrical magnetic bed was filled with a magneto-caloric material, gadolinium, in the form of particles with dimensions ranging from 2 to $5 \mathrm{~mm}$. Halbach array of cylindrical shape was used as magnetic field source”.

\section{PRINCIPLE OF MAGNETIC REFRIGERATION}


The magnetic refrigeration based on magneto-caloric effect. It has two rotating cylinders containing powdered gadolinium - a dense, grey, rare earth metal and a superconducting magnet. Gadolinium has a favorable magneto-caloric coefficient. Each atom of gadolinium has seven unpaired electrons in an intermediate shell, which gives the element a strong magnetic moment. This type of refrigerator is reported to work at a near- room temperature to produce substantial amounts of cooling power. At a fixed temperature, the entropy of a magnetic system gets lowered as the spins align with an applied magnetic field. When a ferromagnetic material, such as gadolinium, is placed in a magnetic field, the magnetic moment of its atoms becomes aligned, making the material more ordered. But, the amount of entropy in the magnet must be conserved, so the atoms vibrate more rapidly, raising the material temperature. Conversely, when gadolinium is taken out of the magnetic field, the material cools. This magneto-caloric effect typically produces a temperature drop of $0.5-2 \mathrm{~K}$ for a field change of 1 Tesla at room temperatures.

The two cylinders containing gadolinium metal can be made to rotate through the magnetic field and arrangements should be made such that, water is pumped into one of the cylinders of gadolinium immediately after it moves out of the magnetic field. The water cools as it flows through the porous bed of demagnetization gadolinium, and then through the heat exchanger. Next, the water passes through the cylinder of gadolinium that is inside the magnetic field. This stream of water heat up and flows through another exchanger, providing ample refrigeration power by continually heating one exchanger and cooling the other. After a preset time interval, the two cylinders of gadolinium compound switch places, and the flow of water is reversed. Besides, antifreeze can be added to the water to allow the machine to cool below zero degrees Celsius.

The four basic processes of conventional gas compression/expansion refrigeration are shown. These are a compression of a gas, extraction of heat, expansion of the gas, and injection of heat. The two process steps extraction of heat and expansion are responsible for a cooling process in two steps. The main cooling usually occurs by the expansion of the gas.

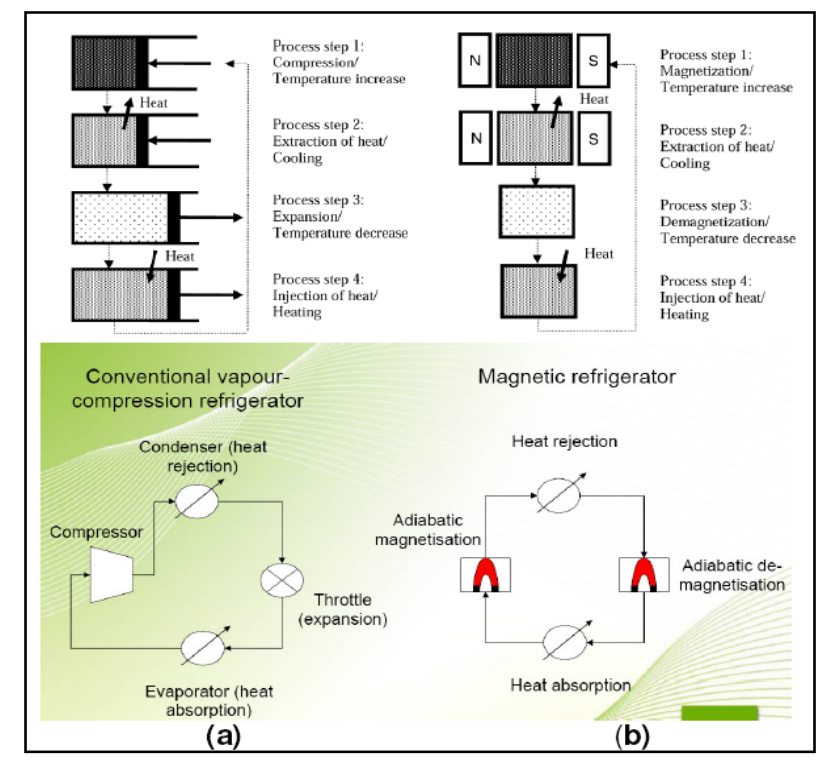

Figure (a): The conventional gas compression process is driven by continuously repeating the four different basic processes shown in this figure. Figure (b): The magnetic refrigeration cycle works analogously. Compression is replaced by adiabatic magnetization and expansion by adiabatic demagnetization.

The magnetic refrigeration process works analogously. By comparing FIG. 1 with FIG. 2 one can see that instead of compression of a gas a magneto caloric material is moved into a magnetic field and that instead of expansion it is moved out of the field. As explained in the previous chapter these processes change the temperature of the material and heat may be extracted, respectively injected just as in the conventional process. 
There is a difference between the two processes. The heat rejection and injection in a gaseous refrigerant is a rather fast process because turbulent motion transports heat very fast and efficient. Unfortunately, this is not the case in the solid magneto caloric materials. Here the transport mechanism for heat is the slow molecular diffusion. Therefore, at present filigree porous structures are considered to be the best solution to overcome this problem. The small distances from center regions of the bulk material to an adjacent fluid domain, where a heat transport fluid captors the heat and transports it away from the material's surface, are ideal to make the magnetic cooling process faster.

\section{MAGNETIC REFRIGERATION CYCLES}

A magnetic refrigeration cycle consists of magnetization and demagnetization in which heat is expelled and absorbed respectively and two other middle processes.

\section{MAGNETIC ERICSSON CYCLE}

1) Isothermal magnetization process I.

2) Iso-field cooling process II

3) Isothermal demagnetization process III

4) Iso-field heating process IV

\section{MAGNETIC BRAYTON CYCLE}

Magnetic Brayton cycle consists of two adiabatic processes and two isofield processes. The magnetic refrigerant cycles between the magnetic field, and the temperature of high and low-temperature heat source. During the isofield cooling process, magnetic refrigerant expels heat. During the isofield heating process, the magnetic refrigerant absorbs heat. No heat flows from and out of the magnetic refrigerant during the adiabatic magnetization process and the adiabatic demagnetization process.

\section{MAGNETIC REFRIGERATION SYSTEM}

Since the lattice entropy of the magnetic refrigerant is large in the range of room temperature and the magnetic entropy change is relatively large only in the vicinity of Curie point, effective entropy change will consequently be small if no measurement is adopted to fetch lattice entropy. In addition, the supply of adequately strong magnetic fields is limited by many factors. As a result, the development of room temperature magnetic refrigeration falls far behind that of cryogenic magnetic refrigeration. However, after Brown constructed the first room temperature magnetic refrigeration system in 1976, researchers all over the world began to lay emphasis on the research on room temperature magnetic refrigeration and developed some guidable magnetic refrigeration systems sequentially.

\section{Brown magnetic refrigerator}

The magnetic refrigerator developed by Brown in 1976 is a reciprocating type and employs an Ericsson like a cycle. The magnetic field is supplied by a water cooled electromagnet, whose maximum strength is $7 \mathrm{~T}$. The magnetic working body immersed in the regenerator consists of $1 \mathrm{~mol}$ Gd plates ( $1 \mathrm{~mm}$ thick) separated by stainless-steel screen wires at an interval of $1 \mathrm{~mm}$ to allow the regenerator fluid to pass in the vertical direction. The adiabatic regenerator is full of a vertical column of fluid $\left(0.4 \mathrm{dm}^{3}, 80 \%\right.$ water, and $20 \%$ alcohol). The working body is held stationary in the magnetic field while the regenerator tube containing the fluid oscillates up and down. The 7-T field is turned on and off at the appropriate time during the cycle to complete the demagnetization cooling, isofield (zero 
fields), magnetization heating and isofield (strong field) processes sequentially. Under the condition of no load, either of the demagnetization and magnetization process deviates from the isothermal process of ideal magnetic Ericsson cycle. And then the temperature span is gradually enlarged with the cycle operation. After about 50 cycles the temperature at the top reaches $46{ }^{\circ} \mathrm{C}$ and the temperature at the bottom reaches $-1^{\circ} \mathrm{C}$, obtaining a global temperature span of $47 \mathrm{~K}$.

\section{Steyert magnetic refrigerator}

An alternative system of a rotating refrigerator in accordance with the Brayton cycle was designed and constructed by Steyert. In this system, a ring-shaped porous magnetic working body rotates across high - field and low - field regions. The heat exchange fluid enters the wheel at a temperature $T_{\text {hot }}$, and leaves the wheel at a temperature $T_{\text {cold }}$ after expels heat to the magnetic refrigerant in low-field. Then, the fluid re-enters the wheel at temperature $\mathrm{T}_{\text {cold }}+\Delta$ after picking up refrigeration heat load $\mathrm{Q}_{\text {cold }}$. In the thermal exchange with the wheel which is at temperature $\mathrm{T}_{\text {hot }}+$ $\Delta$, the fluid warms to $\mathrm{T}_{\text {hot }}+\Delta$. Finally, it deposits heat $\mathrm{Q}_{\text {hot }}$ in the high-temperature heat sink, completing the cycle as it re-enters the wheel at $\mathrm{T}_{\text {hot }}$.

\section{Kirol system}

This system was designed by Kirol as a rotating machine, obeying magnetic Ericsson-like cycle. The magnetic field is supplied with $\mathrm{NdFeB}$ permanent magnet and a maximum $0.9 \mathrm{~T}$ field could be obtained in an air gap. The rotor is constructed of flat disks group of gadolinium with narrow spaces between them. The thickness of each disk is 0.076 $\mathrm{mm}$ and spaces are $0.127 \mathrm{~mm}$ wide. One hundred and twenty-five disks, $270 \mathrm{~g}$ in total weight, are epoxy bonded together to form a rotor. Rectangular flow ports are positioned at magnet edges so that inlet and outlet fluid (water) could flow across the field change regions. Four complete thermodynamic cycles are completed during one circle of the rotor and an $11 \mathrm{~K}$ temperature lift is acquired.

\section{Zimm magnetic refrigerator}

This magnetic refrigerator developed by Zimm is reciprocating type in accordance with the magnetic Brayton cycle. Four processes of the cycle. A maximum $5 \mathrm{~T}$ field is achieved with NbTi superconductor. The concept of AMR cycle is adopted in the regenerator. Approximate $3 \mathrm{~kg}$ Gd spheres, $0.15-0.3 \mathrm{~mm}$ in diameter, are packed in two magnetocaloric beds. The heat transfer fluid is water (added with antifreeze). The results show that in a $5 \mathrm{~T}$ field, it can generate up to $600 \mathrm{~W}$ of cooling power and its efficiency approaches $60 \%$ of Carnot with a COP approaching 15. It can generate about $200 \mathrm{~W}$ cooling power in a $1.5 \mathrm{~T}$ magnetic field. Furthermore, the device has been operated for more than $1500 \mathrm{~h}$ and 18-month period without any major maintenance or any breakdowns.

\section{MAGNETIC REFRIGERATION MATERIALS}

Since Brown first applied ferromagnetic material gadolinium (Gd) in the room temperature magnetic refrigerator in 1976, the research range for magnetic refrigeration working materials has been greatly expanded. At first, some ferromagnets concerning the second order transition were investigated for the large MCE existing in them. Recently the magnetic materials undergoing a first-order magnetic transition become the focus after the giant MCE was found in GdSiGe alloys. The magneto-caloric effect is an intrinsic property of magnetic solid. This effect is obeyed by all transition metals and lanthanide-series elements. The originally suggested refrigerants was a paramagnetic salt, such as cerium magnesium nitrate. Gadolinium, a rare earth metal, exhibits one of the largest known MCE. It was used as a refrigerant for many of the early magnetic refrigeration design. 
Some magnetic materials that promise to be used in the future, the following list of promising categories of magnetocaloric materials for application in magnetic refrigerators:

- Binary and ternary intermetallic compounds

- Gadolinium-silicon-germanium compounds

- Manganites

- Lanthanum-iron based compounds

- Manganese-antimony arsenide

- Iron-manganese-arsenic phosphides

- Amorphous fine met-type alloys (very recent)

\section{ADVANTAGES}

- Purchase cost may be high, but running costs are $20 \%$ less than the conventional chillers. Thus life cycle cost is much less.

- Ozone-depleting refrigerants are avoided in this system, hence it more eco-friendly.

- Energy conservation and reduction in the energy costs.

- The efficiency of magnetic refrigeration is $60 \%$ to $70 \%$.

- Magnetic refrigeration is totally maintenance free \& mechanically simple in construction.

- Noiseless technology(no compressor). This is an advantage in certain contexts such as medical applications.

\section{DISADVANTAGES}

- The initial investment is more as compared with conventional refrigeration.

- The magneto-caloric materials are rare earth materials hence its availability is very difficult.

- Protection of electronic components from magnetic fields. But notice that they are static, of short range and may be shielded.

\section{FUTURE APPLICATIONS}

Some of the future applications are:-

- $\quad$ Magnetic household refrigeration appliances

- Magnetic cooling and air conditioning in buildings and houses

- $\quad$ Central cooling system

- $\quad$ Refrigeration in medicine

- $\quad$ Cooling in food industry and storage

- $\quad$ Cooling in transportation

- $\quad$ Cooling of electronic equipment

\section{CONCLUSIONS}

The permanent magnets and the gadolinium don't require any energy inputs to make them work, so the only energy it takes is the electricity for the motors to spin the wheel and drive the water pumps. Hence the power required to operate this refrigerator is also very less. Magnetic refrigerators have two main advantages over today's 
commercial devices: they do not use hazardous or environmentally damaging chemicals, such as chlorofluorocarbons, and they are up to $60 \%$ efficient. In contrast, the best gas-compression refrigerators achieve a maximum efficiency of about $40 \%$.

- Environmentally safe technology.

- To make the magnetic refrigerator commercially viable, scientists need to know how to achieve larger temperature swings and also permanent magnets which can produce strong magnetic fields of order 10 Tesla.

- Some thermal and magnetic hysteresis problems to be solved for the materials that exhibit the Magneto caloric effect to become really useful.

- No hazardous chemicals used and efficiency of magnetic refrigeration is $60 \%$ to $70 \%$ as compared to Carnot cycle.

\section{ACKNOWLEDGEMENTS}

The authors are grateful to the Maulana Azad National Institute of Technology (M.A.N.I.T) Bhopal for its financial support and for continuous interest in our work.

\section{REFERENCES}

[1] A. Kitanovski, ”Magnetocaloric energy conversion,” Magnetocaloric energy conversion, Switzerland, Springer International publishing, 2015.

[2] A. M. J. Mahdy, ”A novel design of a domestic magnetic refrigerator,” International journal of application or innovation in engineering and management(IJAIEM), pp. 6-19, 2015.

[3] A. Czernuszewicz, J. Kaleta, M. Kro'lewicz, D. Lewandowski*, R. Mech, P.(2013) “A test stand to study the possibility of using magneto caloric materials for refrigerators” Int. J. of refrigeration, 10 Sept. (2013), 1-6.

[4] Y. I. Spichkin ,R.R. Gimaev, (2013) “Experimental methods of the magnetocaloric effect studies” Int. J.of refrigeration,10 Sept. $2013,1-7$.

[5] H. R.E. H. Bouchekara and A Kedous, (2013) “Multiobjective Optimization of AMR System” Int. J. of refrigeration, 3 Sept. 2013,1 -32.

[6] C.R.H. Bahl, K. Engelbrecht, D. Eriksen, K.K. Nielsen, A. Smith, (2013) "Development and experimental results from a 1 kW prototype AMR” Int. J. of refrigeration, 3 Sept. 2013, 1-32.

[7] M. Ozbolt, A. Kitanovski*, J. Tusek, A. Poredos, (2013) “ Electrocaloric Vs. Magnetocaloric energy conversion” Int. J. of refrigeration, 1 July 2013, 1-12.

[8] Peter W. EGOLF*, Andrej KITANOVSKI, Didier VUARNOZ, (2013) “An introduction to Magnetic Refrigeration” 2013.

[9] H. R.E. H. Bouchekara and A Kedous, (2011) “ Electromagnetic design of a magnetic field source for a magnetocaloric refrigerator” Progress in Electromagnetic Research M, 2011 Vol. 19, 251-263.

[10] Engin, Muhammet, Ali and Huseyen.(2009)“Magnetic Refrigeration technology applications on near room temperature.” $5^{\text {th }}$ international advanced technologies symposium (IATS’ 09), May 13 - 15, 2009 Karabuk , Turkey

[11] John , Kurt Roth, and James,(2007)“ Emerging Technology (magnetic refrigeration).” ASHRAE Journal, August 2007, 74-76

[12] T. Utaki, K. Kamiya, and T. Nakagawa. (2007)“ Research on a Magnetic Refrigeration cycle for hydrogen liquefaction.” International Cryocooler Conference, 2007, 645-653

[13] Zimm, C.,(2007) "Design and initial performance of a magnetic refrigerator with a rotating permanent magnet." 2nd International Conference on Magnetic Refrigeration at Room Temperature. et. al. 2007

[14] Andrew, Armando, John and R. Chahine,(2005) “ Near room temperature magnetic refrigeration.” Proceeding of the international green energy conference (IGEC-1) 12-16 June 2005, Paper no. 84, Canada.

[15] B. F. Yu, X. Z. Meng ,Z. Chen. (2003) “ Review on the research of room temperature magnetic refrigeration.” International journal of refrigeration ,26 (2003)622-636, China. 